\title{
Complications of modified Blalock-Taussig shunts mimicking pulmonary disease
}

\author{
M E Coren, C Green, R Yates, A Bush
}

\begin{abstract}
The modified Blalock-Taussig shunt is commonly performed as early palliation in cyanotic congenital heart disease. To highlight unusual diagnostic problems in such patients, two cases are reported in whom shunt complications were initially diagnosed as lobar pneumonia and tuberculosis, respectively. The children, an 8 month old boy and a 3 year old boy, had false aneurysm secondary to infection of the graft and dilatation of the left pulmonary artery caused by blood flow through the shunt, respectively. (Arch Dis Child 1998;79:361-362)
\end{abstract}

Keywords: Blalock-Taussig shunt; congenital heart disease; pulmonary disease

Most infants even with complex congenital heart lesions now survive the neonatal period. In many cases, insertion of a modified BlalockTaussig shunt is performed as a palliative procedure early in life allowing more definitive surgery when the infant has grown. We report

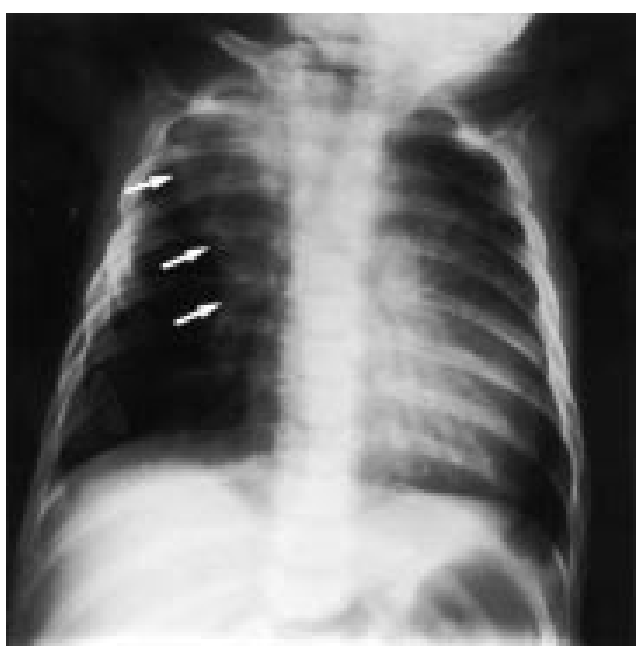

Figure 1 Chest $x$ ray from case 1 showing shadowing in the right upper zone.

Department of Paediatric Respiratory Medicine, The Royal Brompton Hospital, Sydney Street, London SW3 6NP, UK

$M$ E Coren

C Green

R Yates

A Bush

Correspondence to: Dr Bush.

Accepted 8 May 1998

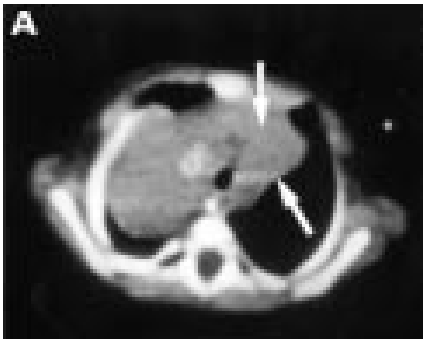
intravenous contrast as the lesion enhances brightly. two children who presented with acute symptoms apparently typical of intercurrent respiratory illness. These cases highlight the additional diagnostic possibilities that exist in patients who have previously undergone cardiac surgery, particularly those with a BlalockTaussig shunt.

\section{Case 1}

An 8 month old boy presented to his local hospital with a history of cough, fever, and poor feeding. He had been born with pulmonary atresia and double outlet right ventricle, and had been treated at 3 months old with a right sided modified Blalock-Taussig shunt. Chest radiography showed right upper lobe shadowing (fig 1) and he was diagnosed with pneumonia. Blood cultures were positive for Streptococcus pneumoniae. The shadowing persisted and he remained pyrexial despite over a week of appropriate intravenous antibiotics and physiotherapy. He was therefore referred to The Royal Brompton Hospital in London, UK where computed tomography revealed a mass lesion in the right upper zone related to the shunt, which was enhanced with intravenous contrast (fig 2). The likely diagnosis was false aneurysm of the Blalock-Taussig shunt secondary to bacterial infection, which was confirmed at thoracotomy when a new shunt was inserted. $\mathrm{He}$ recovered uneventfully and completed six weeks of intravenous antibiotics in his local hospital. He died shortly afterwards of an unrelated viral bronchiolitis.

\section{Case 2}

A 3 year old boy of Bangladeshi origin was admitted for elective cardiac catheterisation to plan further management of his congenital heart disease. He had been treated in infancy with a left sided modified Blalock-Taussig shunt for tetralogy of Fallot and pulmonary atresia. His family reported that he had a persistent cough. Chest radiography (fig 3) showed a mass lesion at the left hilum, thought to be lymphadenopathy, and tuberculosis was suspected. His parents and three siblings were
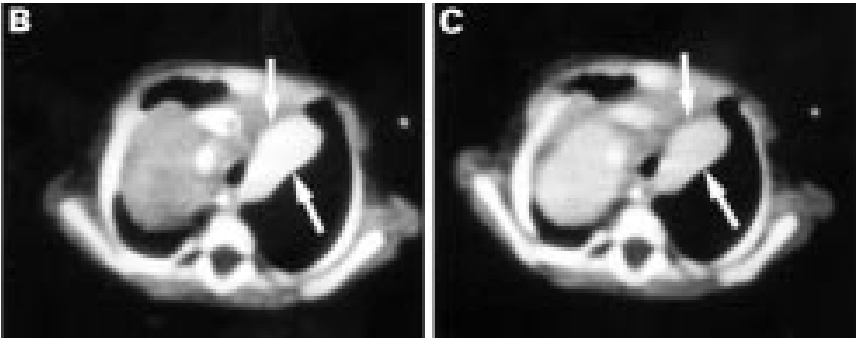

Figure 2 Computed tomography from case 1. (A) A mass lesion in the right chest is seen (arrows). (B) and (C) Effect of 


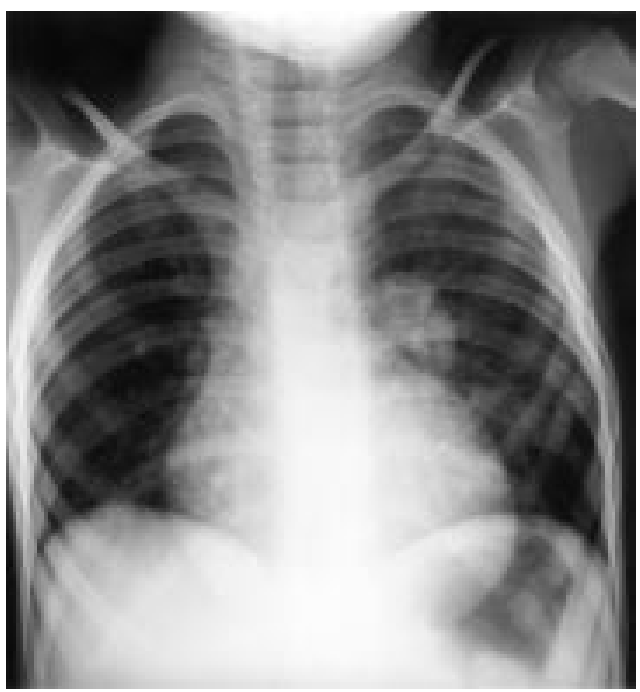

Figure 3 Chest $x$ ray from case 2 showing a mass lesion at the left hilum.

well and he had had no known contact with any cases of tuberculosis. Clinical examination of his chest was normal, Mantoux test was negative and early morning gastric washings failed to show evidence of tuberculosis. Computed tomography revealed aneurysmal dilatation of the left pulmonary artery presumably secondary to blood flow through the shunt. This was confirmed when the planned catheterisation was carried out, before definitive surgery.

\section{Discussion}

The modified Blalock-Taussig shunt, commonly performed as early palliation in cyanotic congenital heart disease, involves insertion of a Gore-tex graft between subclavian and pulmonary arteries to enhance pulmonary blood flow. ${ }^{1}$ In most cases, this allows time for the infant to grow pending a more definitive procedure. ${ }^{12}$ Recognised early complications of these systemic-pulmonary shunts include shunt stenosis and occlusion, nerve damage at the time of operation, and excessive pulmonary blood flow requiring diuretics. ${ }^{2}$ Later problems include progressive inadequacy of the shunt, serous fluid leak, and false aneurysm formation. ${ }^{3}$

At first sight the two children described had classic presentations of common respiratory disease. Case 1 had right upper lobe shadowing and a blood culture positive for a common respiratory pathogen. Case 2, an Asian child, had a cough and a hilar mass suspicious of lymphadenopathy. Both cases were in fact Blalock-Taussig shunt complications, the former a false aneurysm secondary to infection of the graft and the latter dilatation of the left pulmonary artery caused by blood flow through the shunt.

There is a significant number of children who have a modified Blalock-Taussig shunt in situ as a first stage in the treatment of their congenital heart disease. We present these unusual cases to alert clinicians to consider the possibility of a shunt related pathology if such patients present with respiratory symptoms, particularly if there are ipsilateral signs clinically or on chest radiography. Computed tomography with intravenous contrast is a useful mode of investigation.

1 Bove EL, Kohman L, Sereika S, et al. The modified BlalockTaussig shunt: analysis of adequacy and duration of palliation. Circulation 1987;76(suppl III): 19-21.

2 Gladman G, McCrindle BW, Williams WG, Freedom RM, Benson LN. The modified Blalock-Taussig shunt: clinical impact and morbidity in Fallot's tetralogy in the current era. F Thorac Cardiovasc Surg 1997;114:25-30.

3 Sethia B, Pollock JCS. False aneurysm formation: a complication following the modified Blalock-Taussig shunt. Ann Thorac Surg 1986;41:667-8. 